Article

\title{
On the Analysis and Computation of Topological Fuzzy Measure in Distributed Monoid Spaces
}

\author{
Susmit Bagchi \\ Department of Aerospace and Software Engineering (Informatics), Gyeongsang National University, \\ Jinju 660701, Korea; profsbagchi@gmail.com
}

Received: 3 December 2018; Accepted: 20 December 2018; Published: 22 December 2018

\begin{abstract}
The computational applications of fuzzy sets are pervasive in systems with inherent uncertainties and multivalued logic-based approximations. The existing fuzzy analytic measures are based on regularity variations and the construction of fuzzy topological spaces. This paper proposes an analysis of the general fuzzy measures in $n$-dimensional topological spaces with monoid embeddings. The embedded monoids are topologically distributed in the measure space. The analytic properties of compactness and homeomorphic, as well as isomorphic maps between spaces, are presented. The computational evaluations are carried out with $n=1$, considering a set of translation functions with different symmetry profiles. The results illustrate the dynamics of finite fuzzy measure in a monoid topological subspace.
\end{abstract}

Keywords: fuzzy sets; fuzzy measures; monoid topological subspace; compactness; computational applications

\section{Introduction}

The fuzzy set theory has numerous applications in a diverse array of complex systems. Decision making in information systems in the presence of uncertainties or incompleteness can be performed by employing fuzzy sets [1-3]. The inherent uncertainties and imprecision of information can be modeled by employing ordered fuzzy numbers, where the fuzzy ordering is formulated by using trapezoidal structures [4]. In general, the fuzzy decision-making process involves computations on multi-granular rough fuzzy sets [2]. The variations in granularities are reflected in the efficiency of information aggregation and filtering. The convergence theory can be applied in analyzing fuzzy inference techniques with various topological applications. In general, the formation of fuzzy sets and fuzzy topology are constructed based on relational algebra, on a crisp set with an upper limit and lower limit [1].

The hybridizations of fussy sets, lattices, and topology have resulted in the formation of various structures of fuzzy topological spaces with different interpretations. In one approach, the fuzzy set theory is applied to the topology in order to construct fuzzy topological spaces [5]. This formulation has created the structures of $L$-topology based on lattice models. In a different approach, the topological spaces are constructed based on the $L$-subset of the power set of any arbitrary set $X$ [6]. Hence, the topology based on the $L$-subset is called $L$-fuzzy topology, which is a distinct structure as compared to L-topology [7]. An interrelationship exists between the fuzzy sets and the convexity of the underlying spaces. The $L$-convex spaces are formulated based on a continuous and complete lattice [8]. The $L$-convex space supports the embedding of various categories of algebraic structures.

The analysis of compactness of the fuzzy sets and topological spaces is important for formulating corresponding measures [9]. The degree of compactness and countability in L-fuzzy pretopological spaces can be analyzed [10]. The formulation of pretopological spaces is based on the combination of a non-distributive lattice and implication operator algebra. Recently, the graph theoretic view of the 
topology is applied for analyzing the physical lattice-like structures of the honeycomb networks of physical systems [11]. The invariant parameters in the topologically characterized graph-like structures are the topological indices and related connectivity polynomials.

The definitions of the fuzzy topological spaces are refined by incorporating two functors in order to form a category preserving the fuzzy compactness [12]. The concept of the fuzzy ultrametric is introduced based on the sets of probability measures in a supported space [13]. The measure is constructed in a fuzzy metric space with a compact support. The measures of noncompact fuzzy subsets in standard and fuzzy metric spaces are formulated [14]. The regularity of the null-additivity of fuzzy measures on a metric space can be maintained, preserving the consistency of the respective measures [15]. The fuzzy measure on any arbitrary topological space is constructed based on the general Borel sets [16]. The measure preserves the monotone class of the Borel sets and may not be finite in all cases. From the algebraic point of view, the measure on an Abelian group is constructed, which is named as the Haar measure [17]. It is considered that Haar measurable groups are locally compact in nature. The commutative convolution measure algebra is isomorphic to $L^{1}(G)$, with finite dimensional involution representation [18]. If a space is not separable, then an uncountable number of pairwise disjoint subsets exist, which are open and measurable on any locally compact Abelian group [19].

This paper proposes the analysis and computational evaluations of topological fuzzy measures in distributed monoid spaces. It is considered that the underlying space is $n$-dimensional and the distributed embeddings of the monoids are arbitrary in the space. The topological fuzzy measures are finite and can be applied on the subspaces under appropriate translations. The computation of measure is performed in a 1D space under the influence of a set of translation functions with various symmetry profiles. The computational evaluations illustrate that the computed measures exhibit various covering and saturation effects, based on the incorporated beta control of the measure.

The rest of the paper is organized as follows. Section 2 presents the preliminary concepts. Section 3 presents definitions related to the presented model, and Section 4 presents a set of analytical properties. Section 5 presents computational evaluations. Finally, Section 6 concludes the paper.

\section{Preliminaries}

Let $X$ be any arbitrary set equipped with a binary relation (or operation) $*: X^{2} \rightarrow X$. The structure $(X, *)$ is called a group if the following axioms are maintained [20]:

$$
\begin{aligned}
& \forall x, y, z \in X, x *(y * z)=(x * y) * z, \\
& \exists e \in X: \forall x \in X, e * x=x * e=x, \\
& \forall x \in X, \exists x^{-1} \in X: x * x^{-1}=x^{-1} * x=e
\end{aligned}
$$

The group $(X, *)$ is called Abelian if $\forall x, y \in X, x * y=y * x$. If $X$ represents an underlying space, then a corresponding topology $\tau \subseteq P(X)$ can be constructed on it. The topology $\tau$ should maintain the following axioms (I represents the index set) [21]:

$$
\begin{aligned}
& \forall A_{i} \in \tau, i \in I, \cup A_{i \in I} \in \tau, \\
& \forall A_{i}, A_{k} \in \tau, A_{i} \cap A_{k} \in \tau, \\
& \{\phi, X\} \subset \tau
\end{aligned}
$$

A space is called a sigma measurable if it can be equipped with a function, $\mu:\left(A_{i} \subset X\right) \rightarrow[0,+\infty]$. A measure is finite if $\forall A_{i} \subset X, \mu\left(A_{i}\right)<+\infty$. A fuzzy set is a set equipped with a nonbinary discrete measure, given as $\left(X, \mu_{F}\right)$, such that $\mu_{F}: X \rightarrow[0,1]$. A fuzzy set can have a support built into it. 


\section{Definitions}

\subsection{Groupoid Space}

Let $X$ be any arbitrary point set and $A \subset X$ is equipped with an operation in $n$-dimensional space as $o_{x}: A^{2 n} \rightarrow A^{n}$, such that $\forall a, b \in A^{n}, \exists c \in A^{n}$, where $c=a o_{x} b$. The $\left(X^{n}, o_{x}\right)$ is a groupoid space.

\subsection{Distributed Monoid Space}

Let $\left(X^{n}, o_{x}\right)$ be a groupoid space and $M=\left\{B_{i} \subset A^{n}: i \in I \subset Z^{+}\right\}$, where $B_{i} \cap B_{k}=\phi$ if $i \neq k$ and $\cup B_{i \in I}=A^{n}$. The $M$ is a distributed monoid (DM) if $\forall B_{i} \in M$ the following properties are satisfied [20]:

$$
\begin{aligned}
& \forall a, b, c \in B_{i},\left(a o_{x} b\right) o_{x} c=a o_{x}\left(b o_{x} c\right), \\
& \forall a \in B_{i}, \exists e_{i} \in B_{i}: a o_{x} e_{i}=e_{i} o_{x} a=a
\end{aligned}
$$

\subsection{Topological Distributed Monoid (DM)}

Let $\left(X^{n}, o_{x}\right)$ be a groupoid space with a distributed monoid $M$. The $\left(X^{n}, M, \tau\right)$ is a topological DM space if the following topological properties are satisfied in topology $\tau$ on $X^{n}$ :

$$
\begin{aligned}
& \tau \subseteq P\left(X^{n}\right), M \subset \tau, \\
& \tau \backslash M \neq \phi
\end{aligned}
$$

\subsection{Topological Translation}

Let $\left(X^{n}, M, \tau\right)$ be a topological DM space. The $g: X^{n} \rightarrow R$ is a real valued translation function in $\left(X^{n}, M, \tau\right)$, such that $\forall a \in X^{n}, g(a) \in(-\infty, 0) \cup(0,+\infty)$.

\subsection{Fuzzy Topological Measure}

A fuzzy topological measure in $\left(X^{n}, M, \tau\right)$ is defined as $\mu_{\tau}: \tau \rightarrow[0,1]$, such that

$$
\begin{aligned}
& \mu_{\tau}(\phi)=0, \exists \beta_{i} \in(1,+\infty), \\
& \forall D_{i} \in \tau, \mu_{\tau}\left(D_{i}\right)=\frac{1}{\beta_{i}} \sum_{\forall a \in D_{i}}\left|g(a)^{-1}\right|
\end{aligned}
$$

The scaling factor $\beta_{i}$ is a control parameter for the quantization in a fuzzy range for any arbitrary translation function in the topological DM space.

\subsection{Local Isomorphism}

If there is a bijection $f: M \rightarrow M$ in $\left(X^{n}, M, \tau\right)$, such that $\forall B_{i} \in M, \exists B_{k} \in M: B_{i}=f^{-1}\left(B_{k}\right)$, then $f($.$) is a local isomorphism.$

\subsection{Local Homeomorphism}

If there is a bijection $h: M_{x} \rightarrow M_{y}$ in $\left(X^{n}, M_{x}, \tau_{x}\right)$ and $\left(Y^{n}, M_{y}, \tau_{y}\right)$, respectively, such that $\forall B_{i} \in$ $M_{x}, \exists B_{k} \in M_{y}: B_{i}=h^{-1}\left(B_{k}\right)$, then $h($.$) is a local homeomorphism.$

\section{Analytical Properties}

Theorem 1. In $\left(X^{n}, M, \tau\right)$, the topological fuzzy measure in the topological DM subspace is countably additive as $\mu_{\tau}\left(\cup_{i \in I} B_{i}\right)=\sum_{i \in I} \mu_{\tau}\left(B_{i}\right)$.

Proof. Let $\left(X^{n}, M, \tau\right)$ be a topological DM space. Let $\forall B_{i} \in M, \exists \beta_{i} \in(1,+\infty)$, such that $\mu_{\tau}\left(B_{i}\right)=\beta_{i}^{-1} \sum_{\forall a \in B_{i}}\left|g(a)^{-1}\right|$, where $0<\mu_{\tau}\left(B_{i}\right)<1$. As $[i \neq k] \Rightarrow\left[B_{i} \cap B_{k}=\phi\right]$, thus 
$\mu_{\tau}(M)=\sum_{i \in I}\left[\beta_{i}^{-1} \sum_{\forall a \in B_{i}}\left|g(a)^{-1}\right|\right]$. However, in the distributed monoid subspace, $\sum_{i \in I} \mu_{\tau}\left(B_{i}\right)=$ $\sum_{i \in I}\left[\beta_{i}^{-1} \sum_{\forall a \in B_{i}}\left|g(a)^{-1}\right|\right]$. Hence, $\mu_{\tau}\left(\cup_{i \in I}^{\cup} B_{i}\right)=\sum_{i \in I} \mu_{\tau}\left(B_{i}\right)$ in the topological DM space $\left(X^{n}, M, \tau\right)$.

Theorem 2. In the $\left(X^{n}, M, \tau\right)$ space $\exists D, E \in X^{n}$, such that $\mu_{\tau}(D \cap E)=0$ and $\mu_{\tau}(D \cup E) \in(0,1)$.

Proof. Let $\left(X^{n}, M, \tau\right)$ be a topological DM space, such that $\exists D, E \subset X^{n}$, where $\{D, E\} \subset \tau$. Let $\{D, E\} \not \subset \tau \backslash M$ and, by following topological properties, $D \cup E \in \tau$. However, this indicates that $\mu_{\tau}(D \cap E=\phi)=0$ in $\left(X^{n}, M, \tau\right)$. On the other hand, $X^{n} \backslash\{D \cup E\} \neq \phi$ and $\mu_{\tau}(D)+\mu_{\tau}(E)>0$. As $X^{n} \neq \phi$ and $0<\mu_{\tau}\left(X^{n}\right) \leq 1$, thus $\mu_{\tau}(D \cup E)<1$, where $g(.) \in R \backslash\{-\infty, 0,+\infty\}$. Hence, $\mu_{\tau}(D \cup E) \in(0,1)$ in the topological DM space $\left(X^{n}, M, \tau\right)$.

Lemma. In the $\left(X^{n}, M, \tau\right)$ space, the monotonicity of the topological fuzzy measure is preserved as $\mu_{\tau}(\tau)>\mu_{\tau}(M)$.

Proof. In the $\left(X^{n}, M, \tau\right)$ space, $\tau \neq \phi$, and it results in the condition that $\mu_{\tau}(\tau \backslash\{\phi\}) \in(0,1]$. However, $\tau \backslash M \neq \phi$ and $\mu_{\tau}(\tau \backslash M) \in(0,1)$ in the underlying topological DM space. Hence, $\mu_{\tau}(\tau)>\mu_{\tau}(M)$, preserving the monotonicity of the topological fuzzy measure in the $\left(X^{n}, M, \tau\right)$ space.

Theorem 3. In the $\left(X^{n}, M, \tau\right)$ space, the topological fuzzy measure of a monoid is bounded as $\mu_{\tau}\left(\cup_{i \in I} B_{i}\right)<\sum_{i \in I} \mu_{\tau}\left(\overline{B_{i}}\right)$.

Proof. Let $\left(X^{n}, M, \tau\right)$ be a topological DM space, where $\bar{M}=\left\{\overline{B_{i}}: \forall B_{i} \in M\right\}$ and $\overline{B_{i}}=B_{i} \cup$ $\partial B_{i}$. As $M \subset \tau$, thus $\forall B_{i} \in M, \partial B_{i} \subset B_{i}^{c} \subset X^{n}$ in the topological DM space. Hence, $\left[\partial B_{i} \neq \phi\right] \Rightarrow\left[\mu_{\tau}\left(\overline{B_{i}} \in \bar{M}\right)>\mu_{\tau}\left(B_{i} \in M\right)\right]$ in the respective space.

Theorem 4. If $\left(X^{n}, M, \tau\right)$ is a Hausdorff space, then $\exists D, E \in \tau$, such that $\mu_{\tau}(D)=\mu_{\tau}(E)>0$.

Proof. Let $\left(X^{n}, M, \tau\right)$ be a Hausdorff topological DM space and $\exists D, E \in \tau$, such that $D \cap E=\phi$. However, if $|D|=|E|=1$, then $\{D, E\} \subset \tau \backslash M$, where $M \subset \tau$. As the topological translation maintains the property that $\forall a \in D \subset X^{n}: g(a) \in(-\infty, 0) \cup(0,+\infty)$, thus $\mu_{\tau}(D \cup E)>0$ if $D \cup E \neq \phi$. However, if $|D|=|E|=1$ and $a \in D, b \in E$, then $\mu_{\tau}((D \cup E) \backslash\{a, b\})=0$. Hence, $\exists \beta_{D}, \beta_{E}: \beta_{D} \neq \beta_{E}$ and $\beta_{D}, \beta_{E} \in(1,+\infty)$, such that $\mu_{\tau}(D)=\mu_{\tau}(E)>0$.

Theorem 5. In the two Hausdorff topological DM spaces $\left(X^{n}, M_{x}, \tau_{x}\right)$ and $\left(Y^{n}, M_{y}, \tau_{y}\right)$, the monoid subspaces are homeomorphic if $t: Y^{n} \rightarrow X^{n}$ and $\left(h_{o} t\right)\left(M_{y}\right)=\left(h_{o} t\right)^{-1}\left(M_{y}\right)$.

Proof. Let $\left(X^{n}, M_{x}, \tau_{x}\right)$ and $\left(Y^{n}, M_{y}, \tau_{y}\right)$ be two Hausdorff topological DM spaces, and $t: Y^{n} \rightarrow X^{n}$ be any arbitrary function, such that the following property is maintained:

$$
\begin{aligned}
& S=\cup \underset{i \in I}{\cup}\left(B_{i} \in M_{x}\right), \\
& T=\cup_{k \in I}\left(B_{k} \in M_{y}\right), \\
& \forall a \in T, \exists b \in S: a=t^{-1}(b), \\
& {[\{b, c\} \subset S \wedge(b \neq c)] \Rightarrow\left[t^{-1}(b) \neq t^{-1}(c)\right],} \\
& t(T) \subseteq S
\end{aligned}
$$

Furthermore, if $\forall B_{i} \in M_{x}, \exists B_{k} \in M_{y}: t^{-1}\left(B_{i}\right)=B_{k}$, then $t\left(M_{y}\right)=h^{-1}\left(M_{y}\right)$. Hence, $\left(h_{o} t\right)\left(M_{y}\right)=$ $\left(h_{o} t\right)^{-1}\left(M_{y}\right)$ in the $\left(X^{n}, M_{x}, \tau_{x}\right)$ and $\left(Y^{n}, M_{y}, \tau_{y}\right)$ Hausdorff topological DM spaces. 
Theorem 6. In the two Hausdorff topological DM spaces $\left(X^{n}, M_{x}, \tau_{x}\right)$ and $\left(Y^{n}, M_{y}, \tau_{y}\right)$ equipped with $t: Y^{n} \rightarrow X^{n}$, the monoid homeomorphic subspaces are measure equivalent as $\mu_{\tau}\left(t^{-1}(S)\right)=\lambda \mu_{\tau}(h(S))$, where $\lambda>0$ and, $S=\cup_{i \in I}\left(B_{i} \in M_{x}\right)$.

Proof. Let $\left(X^{n}, M_{x}, \tau_{x}\right)$ and $\left(Y^{n}, M_{y}, \tau_{y}\right)$ be two Hausdorff topological DM spaces and $t: Y^{n} \rightarrow X^{n}$, such that $\left(h_{0} t\right)\left(M_{y}\right)=\left(h_{0} t\right)^{-1}\left(M_{y}\right)$. As $S=\cup_{i \in I}\left(B_{i} \in M_{x}\right) \neq \phi$, thus $\mu_{\tau}(S)>0$ in the monoid subspace. This indicates that $\mu_{\tau}(h(S))>0$ as $h(S) \neq \phi$. Let $\exists \lambda>0$, such that $\mu_{\tau}(S)=\lambda \mu_{\tau}(h(S))$. However, as $\left(h_{0} t\right)\left(M_{y}\right)=\left(h_{0} t\right)^{-1}\left(M_{y}\right)$ in the spaces, so $t^{-1}(S)=h(S)$. Hence $\mu_{\tau}\left(t^{-1}(S)\right)=\lambda \mu_{\tau}(h(S))$.

Theorem 7. $\left(X^{n}, M, \tau\right)$ is at least a Haar measurable commutative space if the inverses exists.

Proof. Let $\left(X^{n}, M, \tau\right)$ be a topological DM space. As $M$ is a monoid subspace, thus $\forall B_{i} \in M, \exists e_{i} \in B_{i}$, such that $e_{i} o_{x} E=E$, where $E \subseteq B_{i}$. In the monoid subspace, $\mu_{\tau}\left(e_{i} o_{x} B_{i}\right)=\left[\mu_{\tau}\left(B_{i}\right) \in(0,1)\right]$. However, if $e_{i} o_{x} E=E$, then $E o_{x} e_{i}=e_{i} o_{x} E$ in $M \subset P\left(X^{n}\right)$. Hence, $\left(X^{n}, M, \tau\right)$ is at least a Haar measurable commutative space if $\forall B_{i} \in M, \forall a \in B_{i}, \exists a^{-1} \in B_{i}: a o_{x} a^{-1}=a^{-1} o_{x} a=e_{i}$.

Theorem 8. If $X$ is compact, then $\lim _{i \rightarrow+\infty} \mu_{\tau}\left(\cup_{i \in I} B_{i}\right) \rightarrow 1$.

Proof. Let $X$ be a compact set and $\left(X^{n}, M, \tau\right)$ be a compact topological DM space. If $C$ is a cover of $X^{n}$, then $X^{n} \subseteq \cup_{m \in I}\left(D_{m} \in C\right)$. Let $M \subset C$, such that $\left|X^{n} \backslash \cup_{i \in I} B_{i}\right| \in(0,+\infty)$. This indicates that $\lim _{i \rightarrow+\infty}\left|X^{n} \backslash \cup_{i \in I} B_{i}\right| \rightarrow 0$ in $\left(X^{n}, M, \tau\right)$. However, $0<\mu_{\tau}\left(X^{n} \in \tau\right) \leq 1$ in the respective compact space, where $X^{n} \neq \phi$. Hence, $\lim _{i \rightarrow+\infty} \mu_{\tau}\left(\cup_{i \in I} B_{i}\right) \rightarrow 1$ in the compact $\left(X^{n}, M, \tau\right)$.

Theorem 9. In the $\left(X^{n}, M, \tau\right)$ space $\mu_{\tau}(D)>0$, where $D=\left(\cup_{i \in I} B_{i}\right)^{c}$.

Proof. Let $\left(X^{n}, M, \tau\right)$ be a topological DM space. If $D=\cup_{i \in I}\left(B_{i} \in M\right)$ in the space, then $X^{n} \backslash D \neq \phi$, because $\tau \backslash M \neq \phi$. This indicates that in $\left(X^{n}, M, \tau\right)$, the closed space $D^{c} \neq \phi$, which results in $\mu_{\tau}\left(D^{c}\right)>0$

Theorem 10. In the $\left(X^{n}, M_{x}, \tau_{x}\right)$ and $\left(Y^{n}, M_{y}, \tau_{y}\right)$ locally homeomorphic topological DM spaces, $\sum_{i \in I} \mu_{\tau}\left(B_{i} \in M_{x}\right)=\mu_{\tau}\left(\cup_{k \in I} h^{-1}\left(B_{k} \in M_{y}\right)\right)$.

Proof. Let $\left(X^{n}, M_{x}, \tau_{x}\right)$ and $\left(Y^{n}, M_{y}, \tau_{y}\right)$ be two locally homeomorphic topological DM spaces. Thus, $\forall B_{k} \in M_{y}, \exists B_{i} \in M_{x}: B_{i}=h^{-1}\left(B_{k}\right)$ and $\cup_{i \in I}\left(B_{i} \in M_{x}\right)=\cup_{k \in I} h^{-1}\left(B_{k} \in M_{y}\right)$. This results in $\sum_{i \in I} \mu_{\tau}\left(B_{i} \in M_{x}\right)=\mu_{\tau}\left(\cup_{k \in I} h^{-1}\left(B_{k} \in M_{y}\right)\right)$.

Theorem 11. In the $\left(X^{n}, M_{x}, \tau_{x}\right)$ and $\left(Y^{n}, M_{y}, \tau_{y}\right)$ locally isomorphic as well as homeomorphic topological DM spaces, $\mu_{\tau}\left(\cup_{i \in I}\left(B_{i} \in M_{x}\right)\right)=\mu_{\tau}\left(\cup_{k \in I}\left(h_{o} f\right)^{-1}\left(B_{k} \in M_{y}\right)\right)$.

Proof. Let $\left(X^{n}, M_{x}, \tau_{x}\right)$ and $\left(Y^{n}, M_{y}, \tau_{y}\right)$ be two locally isomorphic as well as homeomorphic topological DM spaces. This indicates that $\forall B_{i} \in M_{x}, \exists B_{u} \in M_{x}: B_{i}=f^{-1}\left(B_{u}\right)$ and $\exists B_{k} \in M_{y}: B_{i}=$ $\left(h_{o} f\right)^{-1}\left(B_{k}\right)$. Thus, in the two topological DM spaces $\underset{i \in I}{\cup}\left(B_{i} \in M_{x}\right)=\cup_{k \in I}\left(h_{o} f\right)^{-1}\left(B_{k} \in M_{y}\right)$, which results in $\mu_{\tau}\left(\cup_{i \in I}\left(B_{i} \in M_{x}\right)\right)=\mu_{\tau}\left(\cup_{k \in I}\left(h_{o} f\right)^{-1}\left(B_{k} \in M_{y}\right)\right)$.

Theorem 12. The topological fuzzy measure is consistent under compression if $\forall D_{i} \in \tau \backslash\{\phi\}, \beta_{s}>$ $\sup \left(\left\{\beta_{i}: D_{i} \in \tau \backslash\{\phi\}\right\}\right)$ and, $\mu_{\tau}\left(D_{i}\right)=\frac{1}{\beta_{s}} \sum_{\forall a \in D_{i}}\left|g(a)^{-1}\right|$. 
Proof. Let $\left(X^{n}, M, \tau\right)$ be a topological DM space equipped with fuzzy measure $\mu_{\tau}($.$) . The translation$ function maintains $\forall a \in X^{n},\left|g(a)^{-1}\right| \in(0,+\infty)$. Let the topological DM space be $\forall D_{i} \in \tau, \beta_{s}=$ $\sup \left(\left\{\beta_{i}: D_{i} \in \tau \backslash\{\phi\}\right\}\right)+(\varepsilon>0)$, where $\mu_{\tau}\left(D_{i}\right) \in[0,1]$. As a result, the following condition will be maintained by compressed measure:

$$
\begin{aligned}
& \forall D_{i} \in \tau \backslash\{\phi\}, \\
& \frac{1}{\beta_{s}} \sum_{\forall a \in D_{i}}\left|g(a)^{-1}\right|<\frac{1}{\beta_{i}} \sum_{\forall a \in D_{i}}\left|g(a)^{-1}\right|
\end{aligned}
$$

However, $\forall D_{i} \in \tau \backslash\{\phi\}, \mu_{\tau}\left(D_{i}\right) \in(0,1]$ in $\left(X^{n}, M, \tau\right)$.

Hence, $0<\frac{1}{\beta_{s}} \sum_{\forall a \in D_{i}}\left|g(a)^{-1}\right|<1$, maintaining consistency under compression.

\section{Computational Evaluations}

The computational evaluations are carried out in a 1D topological DM space with distributed monoid embedding, such that $\forall a \in X \subset R, a \in(-\infty,+\infty)$. The computational model of measure in the distributed monoid considers, $M=\left\{\left(A \subset X, o_{x}\right)\right\}$, where $A=\{a: \forall a \in[-1,1] \backslash\{0\}\}$, such that $\forall a, b \in A: a o_{x} b \Rightarrow[a . b \in A]$. The measures are numerically computed by employing translation functions having various profiles, such as (a) inverse translation $\left(g(a)^{-1}=a^{-1}\right)$, (b) fixed coefficient translation $\left(g(a)^{-1}=k a, k \in R^{+}\right)$, and (c) nonlinear periodic translation $\left(g(a)^{-1}=\cos ^{-1}(k a) \neq 0\right)$ in a topological DM space. The numerical datasets are generated by software written in $C$ language with installed Math-libraries. The samples are collected in datasets covering entirely positive and negative domains. The resulting profiles of the translation functions of different categories with various symmetries are presented in Figures 1-3.

The profile of inverse translation, presented in Figure 1, illustrates that the translation has a higher concentration zone around the origin, and appears to have symmetric distribution. The fixed coefficient translation profiles presented in Figure 2 consider two coefficient values, and the resulting profiles illustrate that the variations in translations have approximately symmetric distributions in the domain. The inverse and fixed coefficient translations do not cover a null (zero) translation point (i.e., translations are always in the non-zero positive range). The profile of the nonlinear periodic translation is presented in Figure 3, which is asymmetric in nature, with a monotonic reduction of translation in the domain. Moreover, the nonlinear periodic translation covers a null (zero) translation point (i.e., preserving translation invariant fixed point). The variations of the aggregate (sum) of translations covering $M=\left\{\left(A, o_{x}\right)\right\}$ in different cases are presented in Figure 4.

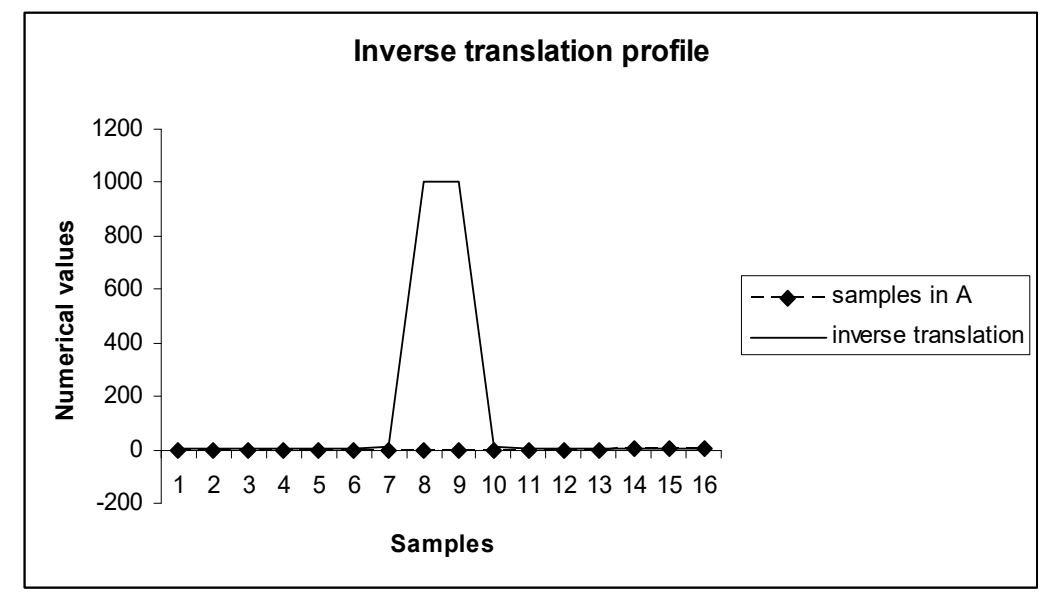

Figure 1. Profile of modulus of inverse translation. 


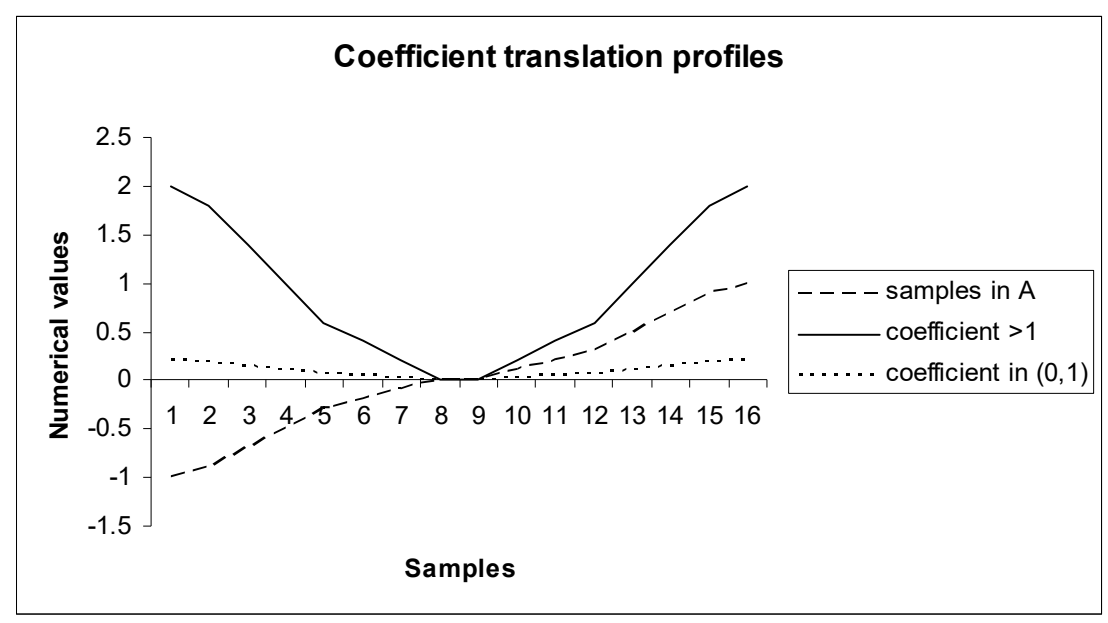

Figure 2. Profiles of modulus of multiplicative translation.

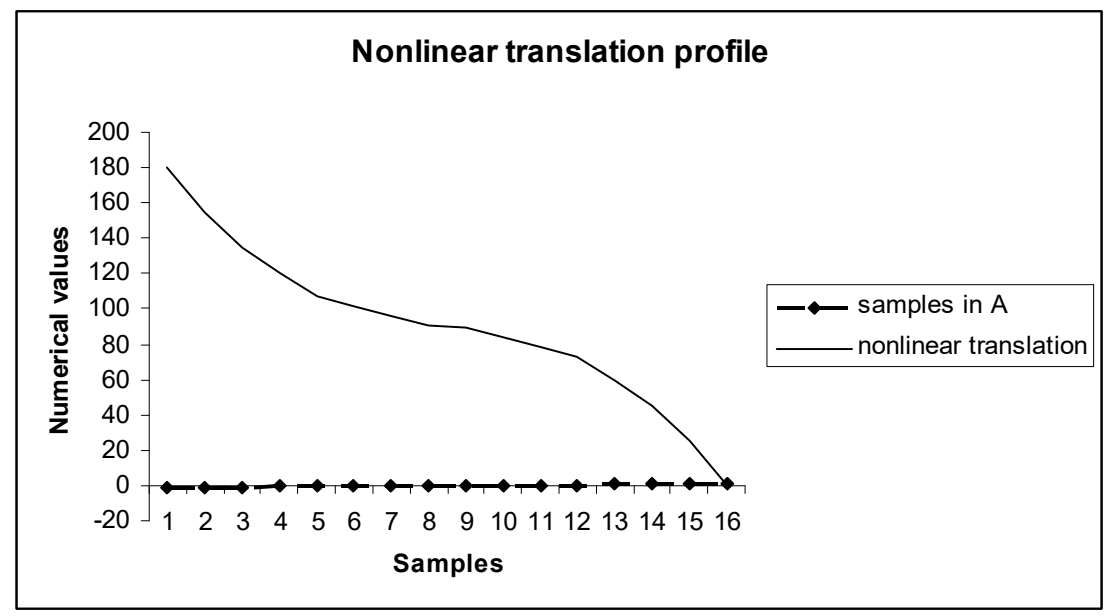

Figure 3. Profile of modulus of nonlinear trigonometric translation.

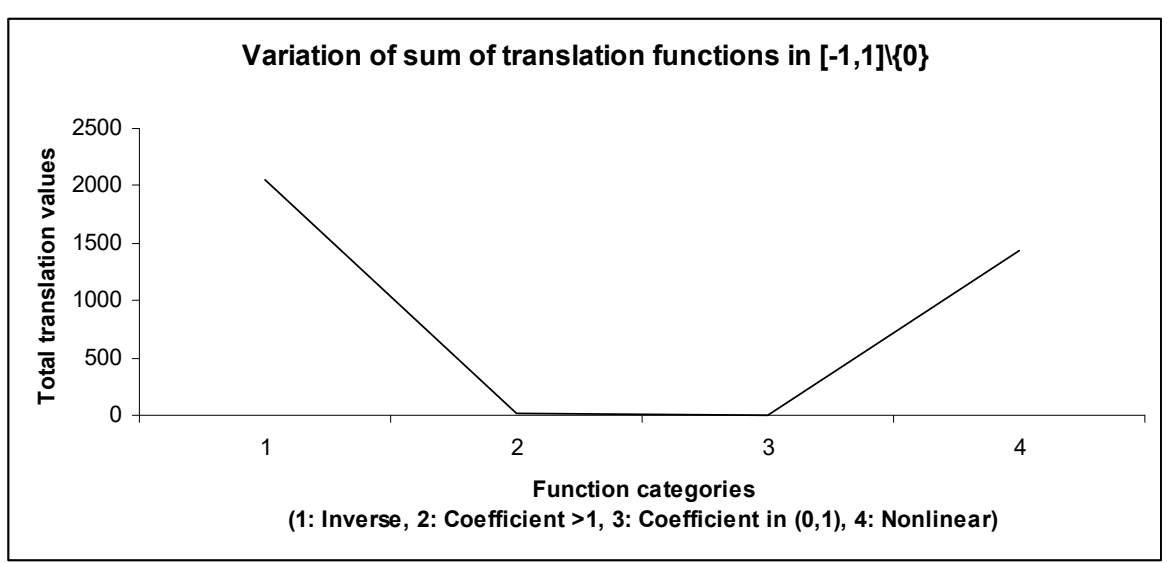

Figure 4. Variations of aggregate functional translation for different categories.

The aggregated translation is much smaller in case of the fixed coefficient translations as compared to the other categories of translations. The inverse translation has the highest aggregated value because of the existence of a singularity at the origin. However, the nonlinear periodic translation exhibits a moderately higher aggregated translation value.

The fuzzy measures are computed considering two cases, such as (1) deterministic measures under $\sup ($.$) control, and (2) random measure under the control of randomized \beta_{i}$ values maintaining 
conditions of fuzzy measures. The dynamics of the resulting fuzzy measures are presented in Figures 5 and 6.

Fuzzy measure under sup(.) control
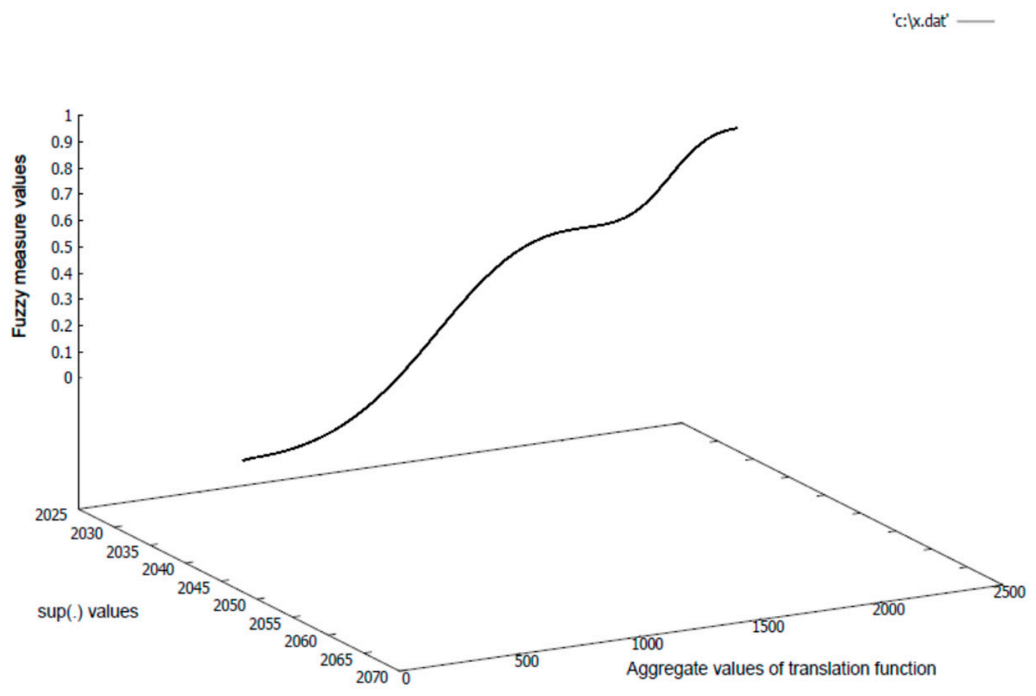

Figure 5. Variation of fuzzy measure under constant sup(.) control.

Fuzzy measure under varying beta control

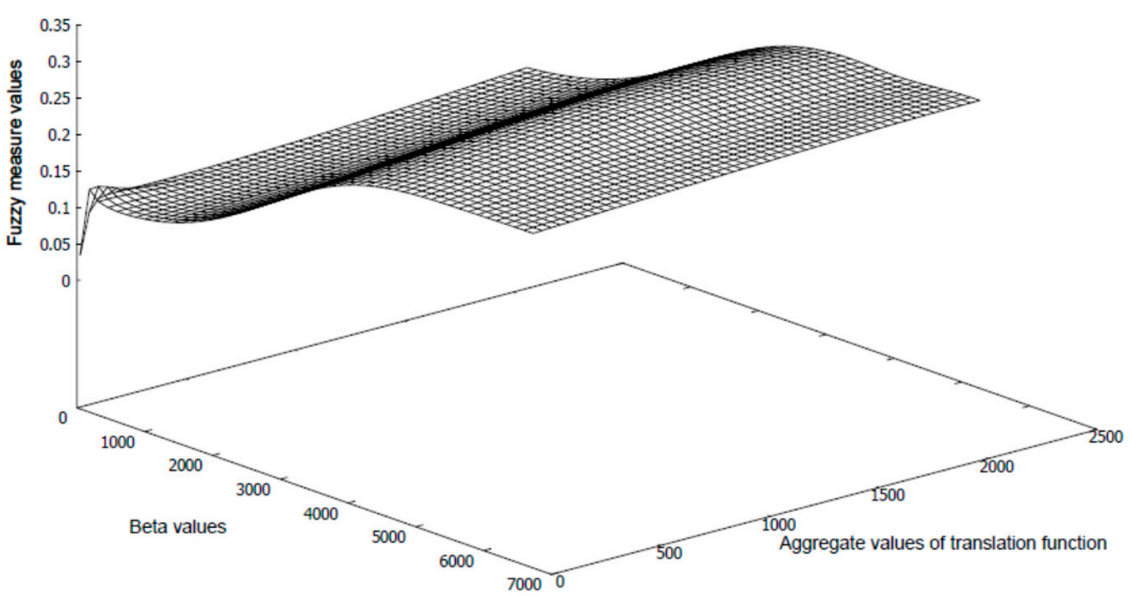

Figure 6. Surface map of fuzzy measure under randomly varying beta control.

The sup(.) control considers a fixed beta value as a control parameter, based on a set of translation values, and it exerts a compressive effect on the distribution of measures. As a result, the domain of distribution of the fuzzy measure tends to cover $[0,1]$ entirely, as presented in Figure 5. Moreover, the covering measure distribution is nonlinear in nature. However, the randomized fuzzy measures are computed based on randomly generated beta control values, depending on the respective individual translation profiles. As a result, it generates a nonlinear measure surface with a saturation effect. The saturation effect depends upon two factors, namely (1) randomized distribution of the $\beta_{i}$ control parameter and (2) variations in the localized random measures based on distinctive individual $\beta_{i}$ values. The variations of the $\beta_{i}$ control parameters are presented in Figure 7. As the randomized control parameter is monotonically increased, the aggregated translation values incorporate saturation 
at a comparatively lower limit boundary $(<1)$. However, both of the fuzzy measures are consistent in nature within the space.

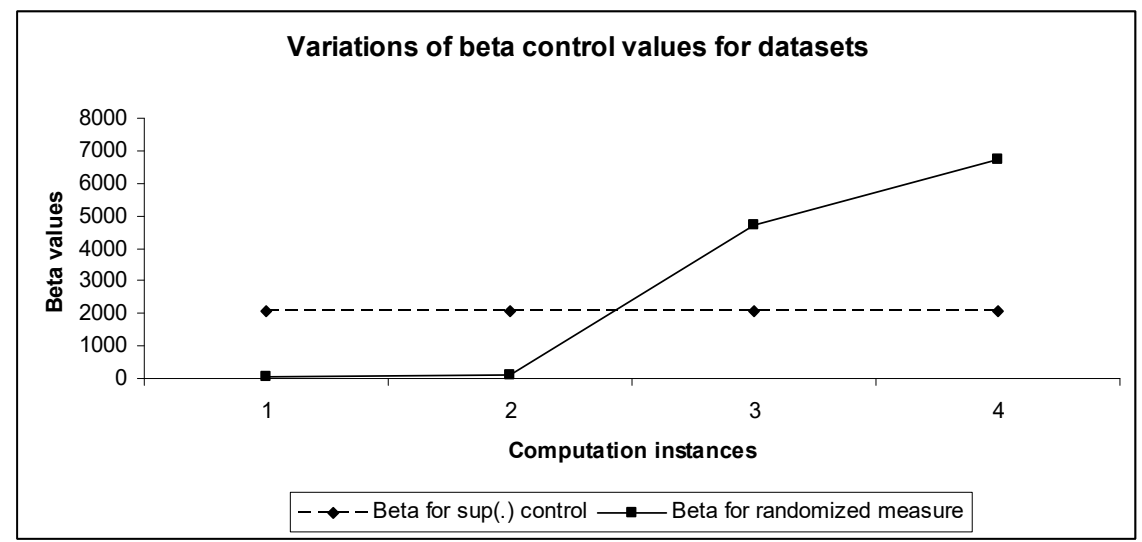

Figure 7. Variations of beta control values for various datasets.

\section{Conclusions}

The analytic understanding of topological fuzzy measures facilitates the construction of specific measure spaces along with its applications. The analysis and computation of topological fuzzy measures vary if the underlying spaces embed monoid algebraic structures. In this paper, the concept of topologically distributed monoid spaces is presented, and a set of novel theorems is constructed in order to gain analytical insights into fuzzy measures in such spaces. The fuzzy measure of the distributed monoid spaces can be computed with diverse distribution profiles covering the entire measure range, using a specific control parameter. The measures are finite in nature and the equivalent topological fuzzy measures in two locally homeomorphic monoid spaces can be formulated if the underlying space is Hausdorff. The proposed topological fuzzy measure is consistent under compression. In compact spaces, the covering topological fuzzy measure of the distributed monoids preserves the limiting unity measure. Furthermore, the minimal form of the fuzzy Haar measurability can be preserved in the topological distributed monoid spaces under certain conditions. The minimal Haar measurability of the proposed topological fuzzy measure considers the identity elements only. As a limiting case, the proposed measure does not consider the existence of a group structure in the underlying space. The topological fuzzy measures in the distributed monoid spaces can be computed under various translations, generating a variety of measure distributions and covers.

Funding: The funding of this work is partly covered by Gyeongsang National University, Jinju, ROK.

Acknowledgments: The author would like to thank the editors and anonymous reviewers for their helpful comments and suggestions.

Conflicts of Interest: The author declares no conflict of interest.

\section{References}

1. Chen, B. $\beta$-Convergence theory of nets of fuzzy sets in fuzzy topological spaces. In Proceedings of the International Conference on Machine Learning and Cybernetics, Hebei, China, 12-15 July 2009.

2. Xue, Z.A.; Han, D.J.; Lv, M.J.; Zhang, M. Novel three-way decisions models with multi-granulation rough intuitionistic fuzzy sets. Symmetry 2018, 10, 662. [CrossRef]

3. Liu, D.; Chen, X.; Peng, D. Cosine distance measure between neutrosophic hesitant fuzzy linguistic sets and its application in multiple criteria decision making. Symmetry 2018, 10, 602. [CrossRef]

4. Piasecki, K.; Hanckowiak, A.L. On approximation of any ordered fuzzy number by a trapezoidal ordered fuzzy number. Symmetry 2018, 10, 526. [CrossRef]

5. Chang, C.L. Fuzzy topological spaces. J. Math. Anal. Appl. 1968, 24, 182-190. [CrossRef] 
6. Höhle, U. Upper semicontinuous fuzzy sets and applications. J. Math. Anal. Appl. 1980, 78, 659-673. [CrossRef]

7. Fang, J. Sums of L-fuzzy topological spaces. Fuzzy Sets Syst. 2005, 157, 739-754. [CrossRef]

8. Jin, Q.; Li, L. On the embedding of convex spaces in stratified L-convex spaces. Springer Plus 2016, 5, 1610. [CrossRef] [PubMed]

9. Shi, F.G. A new definition of fuzzy compactness. Fuzzy Sets Syst. 2007, 158, 1486-1495. [CrossRef]

10. Shi, F.G.; Liang, C. Measures of compactness in L-fuzzy pretopological spaces. J. Intell. Fuzzy Syst. 2014, 26, 1557-1561.

11. Hussain, Z.; Munir, M.; Rafique, S.; Kang, S.M. Topological characterizations and index-analysis of new degree-based descriptors of honeycomb networks. Symmetry 2018, 10, 478. [CrossRef]

12. Lowen, R. Fuzzy topological spaces and fuzzy compactness. J. Math. Anal. Appl. 1976, 56, 621-633. [CrossRef]

13. Savchenko, A.; Zarichnyi, M. Fuzzy ultrametrics on the set of probability measures. Topology 2009, 48, 2009. [CrossRef]

14. Ban, A.I.; Gal, S.G. Measures of noncompactness for fuzzy sets in fuzzy topological spaces. Fuzzy Sets Syst. 2000, 109, 205-216. [CrossRef]

15. Song, J.; Li, J. Regularity of null-additive fuzzy measure on metric spaces. Int. J. Gen. Syst. 2003, 32, $271-279$. [CrossRef]

16. Wu, J.; Wu, C. Fuzzy regular measures on topological spaces. Fuzzy Sets Syst. 2001, 119, 529-533. [CrossRef]

17. Rudin, W. Measure algebras on abelian groups. Bull. Am. Math. Soc. 1959, 65, 227-247. [CrossRef]

18. Albar, S.F. Noncommutative convolution measure algebras with no proper L-ideals. Bull. Aust. Math. Soc. 1989, 40, 13-23. [CrossRef]

19. Ohrysko, P.; Wojciechowski, M.; Graham, C. Non-separability of the Gelfand space of measure algebras. Ark. Mat. 2016, 54, 525-535. [CrossRef]

20. Herstein, I.N. Abstract Algebra, 3rd ed.; Wiley: Hoboken, NJ, USA, 1996.

21. Burgess, D.C.J. Analytical Topology, 1st ed.; Van Nostrand Reinhold Inc.: New York, NY, USA, 1966.

(C) 2018 by the author. Licensee MDPI, Basel, Switzerland. This article is an open access article distributed under the terms and conditions of the Creative Commons Attribution (CC BY) license (http:/ / creativecommons.org/licenses/by/4.0/). 